\title{
Aprendizado do uso do inalador dosimetrado após explicação por pneumologista*
}

\author{
Alessandra SANDRINI ${ }^{1}$, ANDRÉIA JACOMOSSI ${ }^{2}$, SONIA MARIA FARENSIN ${ }^{3}$, \\ ANA LUISA GODOY FERNANDES ${ }^{4}$, JOSÉ ROBERTO JARDIM ${ }^{4}$
}

Introdução: O uso do inalador dosimetrado para administração de drogas inalatórias tem sido preconizado há vários anos. Apesar disso, um grande número de pacientes deixa de ser tratado por essa via de administração. Médicos deixam de prescrever drogas inalatórias por esse dispositivo por acreditar que os pacientes serão incapazes de realizar a técnica corretamente. Por outro lado, os médicos não despendem tempo apropriado para ensinar a técnica correta de uso dos inaladores dosimetrados. Objetivo: Avaliar a percentagem de pacientes, nunca ensinados a usar inalador dosimetrado, que aprendem a utilizar corretamente este dispositivo, após explicação fornecida por pneumologista. Material e métodos: Foram estudados, prospectivamente, 119 pacientes em uma clínica privada em São Paulo. Os pacientes eram ensinados exaustivamente a utilizar o dispositivo na primeira consulta e orientados a retornar em dez dias, quando eram solicitados a realizar a técnica exatamente como estavam fazendo no domicílio. Foi classificada em quatro categorias a técnica de uso: correto, pouco errado, erro intermediário, muito errado. Resultados: Foram excluídos 26 pacientes, devido ao não comparecimento à consulta de retorno no tempo previsto. A amostra resultou em 93 pacientes. A idade dos pacientes variou de 9 a 81 anos, com média de 42,6 ( \pm 21$)$ anos; $59,2 \%$ eram do sexo masculino e $40,8 \%$ do feminino. Após dez dias, 45 pacientes $(48,4 \%)$ realizaram a técnica correta. Usaram de forma totalmente errada o inalador dosimetrado $16,2 \%$ dos pacientes; $19,3 \%$ apresentaram técnica pouco errada e 16,2\%, erro intermediário. Os erros mais

freqüentes foram: colocação do dispositivo dentro da boca; inspiração antes de acionar o jato; inspiração rápida; inspiração pelo nariz. Apenas 33,3\% dos pacientes abaixo de 15 anos e acima de

75 anos souberam realizar a técnica de forma totalmente correta. Conclusão: Este estudo demonstrou que a maioria dos pacientes aprende a usar corretamente ou quase corretamente a medicação por esse dispositivo após uma primeira explicação, desde que exaustivamente ensinada.

(J Pneumol 2000;27(1):7-10)

\section{Metered-dose inhaler technique learning after explanation given by pulmonologist}

\begin{abstract}
Introduction: The use of inhaled drugs through metered-dose inhaler has been advocated for years. Nonetheless, a small number of patients are routinely treated by this technique.

Prescriptions of inhaled drugs by healthcare providers are not frequent, usually because they anticipate patients will not be able to perform the technique correctly. In addition, healthcare providers do not usually take their time to appropriately teach patients how to use metered-dose inhalers correctly. Objective: To evaluate the percentage of uninstructed patients that learn how to use a metered-dose inhaler correctly after being taught by a pneumologist. Patients and methods: One hundred and nineteen patients from a private clinic were prospectively studied in São Paulo, southeastern Brazil. Patients were exhaustively taught how to use the metered-dose inhaler at the first consultation and were told to return after 10 days, when they were asked to
\end{abstract}

* Trabalho realizado na Universidade Federal de São Paulo.

1. Pneumologista Pós-graduanda da Disciplina de Pneumologia.

2. Ex-Estagiária da Disciplina de Pneumologia.

3. Professora da Disciplina de Pneumologia.

4. Professor-Adjunto da Disciplina de Pneumologia.
Endereço para correspondência - José Roberto Jardim, Rua Botucatu, $740-3^{\circ}$ andar (Pneumologia) - 04023-062 - São Paulo, SP. Tel. (11) 576-4238; Fax (11) 575-7424; E-mail: josejardim@pneumo.epm. br

Recebido para publicação em 10/12/99. Aprovado, após revisão, em 18/9/00. 
perform the technique exactly the way they were doing it at home. Their performance was classified as correct, slightly incorrect, intermediately incorrect, and totally incorrect. Results: Twenty-six patients were excluded for not coming to the second appointment. The final sample comprised 93 patients. Age ranged from 9 to 81 years (mean age $42.6 \pm 21$ ); 59.2\% of the patients were male and $40.8 \%$ female. Considering all patients, only 16.25 of them used the metered-dose inhaler totally incorrectly and $19.3 \%$ performed the technique intermediately incorrectly. The most frequent mistakes were: metered-dose inhaler device was placed inside the mouth; inspiration before jet was activated; fast aspiration; inspiration through the nose. Thirty-

three percent of patients who were under 15 and over 75 years performed the technique correctly. Conclusion: This study has demonstrated that the majority of the patients learned how to use the metered-dose inhaler correctly after being properly and extensively taught.

Descritores - Nebulizadores e vaporizadores. Administração por inalação. Aprendizagem.

Key words - Nebulizers and vaporizers. Inhalation administration. Learning.

\section{INTRODUÇÃO}

O tratamento local do trato respiratório com drogas por via inalatória tem sido usado por muitos anos na asma. Os inaladores dosimetrados são utilizados desde 1950 e os inaladores de pó seco surgiram na década de 70. As vantagens da rota inalatória são muitas, incluindo exigência de pequena quantidade de droga para obtenção de efeito significativo, menor incidência de efeitos colaterais $e$ início rápido de ação(1). Apesar disso, muitos pacientes que necessitam de medicação inalatória deixam de utilizar esta via de administração em decorrência da dificuldade da realização da técnica correta de uso do dispositivo, principalmente dos inaladores dosimetrados. Com o aparecimento das câmaras de expansão, esse problema foi resolvido parcialmente. A câmara de expansão (espaçador) permite o uso da medicação por inalador dosimetrado sem exigir do paciente a correta coordenação dos movimentos no momento do acionamento do jato. Entretanto, muitos pacientes resistem ao uso de câmaras de expansão por razões de comodidade e até mesmo de estética.

O aprendizado do uso da medicação por inalador dosimetrado depende do grau de compreensão do paciente e do tempo de que o médico, ou algum outro profissional da área de saúde, dispõe para ensinar-lhe a técnica correta. Baseados nisso, objetivamos estudar a percentagem de pacientes que aprendem a usar corretamente a medicação por inalador dosimetrado, sem câmara de expansão, após uma explicação dada pelo médico em clínica privada.

\section{MATERIAL E MÉTODOS}

Foi estudada, prospectivamente, uma amostra de 119 pacientes com doença pulmonar obstrutiva que nunca haviam feito uso de medicação inalatória por inalador dosimetrado, provenientes de uma clínica privada na cidade de São Paulo. A seleção das fichas de atendimento dos pacientes foi seqüencial durante um período de oito meses.

Todos os pacientes eram ensinados pelo médico a utilizar a medicação inalatória com inalador dosimetrado na primeira consulta, tantas vezes quantas necessárias, até que aprendessem a técnica corretamente. Nenhum paciente havia recebido qualquer orientação prévia quanto ao uso dessa via de administração de medicação. Os pacientes foram instruídos, diretamente pelos médicos, sem auxílio de material visual, a posicionar o dispositivo na posição vertical, $3-4 \mathrm{~cm}$ afastados dos lábios; a abrir a boca e acionar o jato no início de uma inspiração lenta e profunda; sustentar a respiração por dez segundos; expirar pela boca. Os pacientes eram orientados a retornar dez dias após a primeira consulta. Todos eram instruídos a agitar o dispositivo antes do seu uso para a completa mistura do medicamento e do gás. Na consulta de retorno, eram solicitados a repetir a técnica da forma a que procediam no domicílio.

A classificação da técnica de uso do inalador dosimetrado foi feita da seguinte forma: correto: ausência de qualquer erro na técnica de uso do inalador dosimetrado; pouco errado: dispositivo posicionado dentro da boca ou a mais de $5 \mathrm{~cm}$ de distância ou inspiração rápida ou apnéia curta; erro intermediário: associação de dois erros descritos na categoria anterior; muito errado: inspiração pelo nariz e/ou insuflação pulmonar antes do acionamento, ou mais de dois erros descritos acima.

Foram calculadas as freqüências (relativa e absoluta) dos pacientes de acordo com a classificação da técnica de uso do inalador dosimetrado na consulta de retorno.

\section{REsUlTADOS}

Foram ensinados 119 pacientes com obstrução crônica ao fluxo aéreo (asma ou doença pulmonar obstrutiva crônica), que necessitavam de uso de broncodilatador por via inalatória. Foram excluídos 26 pacientes, devido ao 
não comparecimento à consulta de retorno no tempo previsto. A amostra resultou em 93 pacientes. A idade variou de nove a 81 anos, com média de 42,6 ( \pm 21 ) anos. Dos 93 pacientes, $59,2 \%$ eram do sexo masculino $e$ $40,8 \%$ do feminino. Houve uso de técnica correta em 45 pacientes $(48,4 \%)$, considerando-se todas as faixas etárias. Apenas $16,2 \%$ dos pacientes usaram o inalador dosimetrado de forma totalmente errada; $19,3 \%$ apresentaram técnica pouco errada e $16,2 \%$, erro intermediário (Tabela 1). Os erros mais freqüentes foram: colocação do dispositivo dentro da boca (28 pacientes, 68\%); inspiração antes de acionar o jato (sete pacientes, 15,5\%); inspiração rápida (cinco pacientes, $11 \%$ ); inspiração pelo nariz (quatro pacientes, 9\%). Em relação à faixa etária, os pacientes abaixo de 15 anos e os acima de 75 anos concentraram maior freqüência de técnica incorreta; apenas $33,3 \%$ dos pacientes nessa faixa etária souberam realizar a técnica de forma totalmente correta. Em ambos os sexos a percentagem de aprender a usar o dispositivo de forma correta foi semelhante.

\section{DISCUSSÃO}

A administração de droga por via inalatória, tanto na asma como na doença pulmonar obstrutiva crônica, apresenta inúmeras vantagens sobre as outras vias. $\mathrm{O}$ início de ação de broncodilatadores, por exemplo, é extremamente rápido, ocorrendo dentro dos primeiros dois minutos e atingindo broncodilatação máxima em 15 minutos, resultando em aumento de até $30 \%$ no $\mathrm{VEF}_{1}$ basal pré-broncodilatador em asmáticos ${ }^{(2)}$. Além da rapidez da ação do medicamento, a necessidade de menor dose de princípio ativo com conseqüente menor índice de efeitos colaterais coloca a medicação por via inalatória como mandatória no manejo desses pacientes ${ }^{(3)}$. O uso dos inaladores dosimetrados vem sendo preconizado desde o seu aparecimento, em 1956. Estes dispositivos são leves, fáceis de transportar, manusear e limpar, proporcionam bom aporte pulmonar e requerem manobra inspiratória de baixo fluxo para correta penetração da droga. O baixo custo constitui uma vantagem importante em nosso meio

TABELA 1

Aprendizado do uso do inalador dosimetrado em 93 pacientes com doença pulmonar obstrutiva, dez dias após terem sido exaustivamente ensinados $(42,6 \pm 21$ anos $)$

\begin{tabular}{lc}
\hline \multicolumn{1}{c}{ Técnica } & $\mathbf{N}^{\mathbf{0}}$ de pacientes $\mathbf{( \% )}$ \\
\hline Correta & $45(48 \%)$ \\
Não correta & $48(52 \%)$ \\
Muito errado & $15(16,2 \%)$ \\
Erro intermediário & $15(16,2 \%)$ \\
Pouco errado & $18(19,3 \%)$ \\
\hline
\end{tabular}

em relação aos dispositivos de pó seco. Apesar das características favoráveis ao seu uso, uma das grandes dificuldades em obter altos índices de pacientes utilizando medicação por essa via inclui o preconceito entre os pacientes e, curiosamente, entre médicos. Uma das causas da resistência ao uso dos inaladores dosimetrados por parte dos pacientes é a dificuldade no aprendizado da técnica correta, uma vez que dependem dela a segurança e a eficácia da medicação(4). Em decorrência disso, muitos profissionais optam por não administrar a medicação antes mesmo de fazer uma tentativa inicial. Interiano $e$ Guntupalli(i) observaram que existe grande percentagem de erro na realização da técnica do uso do inalador dosimetrado entre profissionais da saúde, chegando a $96 \%$ entre enfermeiras em seu estudo. Os pneumologistas que erraram, o fizeram de forma parcial e em taxas mais baixas $(15 \%)$.

No nosso estudo, foi observado que $67,7 \%$ dos pacientes aprenderam a usar a medicação por inalador dosimetrado de forma totalmente correta ou minimamente errada após uma única sessão de explicação. Oliveira et al. ${ }^{(6)}$ obtiveram incidência semelhante à dos nossos resultados de uso correto do inalador dosimetrado entre pacientes asmáticos de baixa renda, ao serem inseridos em um programa educacional em asma, conduzido no Brasil. Apenas $16 \%$ dos pacientes em nosso estudo usaram a medicação com técnica totalmente errada na consulta de retorno. Estes dados são semelhantes aos de pacientes asmáticos de mesma faixa etária de um hospital público (dados pessoais não publicados).

Na população deste hospital público, não foi encontrada diferença significativa quanto ao aprendizado do uso da medicação inalatória por inalador dosimetrado em relação aos pacientes da clínica privada, que poderiam apresentar maior facilidade no aprendizado, supostamente por ter melhor nivel sociocultural. Estes resultados contrapõem-se aos obtidos por Epstein et al. ${ }^{(7)}$, que demonstraram que apenas $10,8 \%$ de 130 pacientes realizaram corretamente os 11 passos recomendados para o uso correto do inalador dosimetrado. Nesse estudo, foram avaliados pacientes em uso de inaladores dosimetrados que eram encaminhados rotineiramente para realização de espirometria. No laboratório de função pulmonar, os pacientes eram solicitados a usar o inalador como o faziam no domicílio. Nenhuma explicação era dada pelos investigadores, antes da realização da técnica pelos pacientes. Os pacientes aplicavam o que haviam aprendido quando receberam a prescrição do dispositivo, incluindo explicações dadas por médicos, enfermeiros e mesmo pessoas leigas. Dessa forma, essa pode ser uma explicação para a diferença dos resultados em relação ao nosso estudo. Shim e Williams ${ }^{(8)}$ demonstraram que $53 \%$ de 30 pacientes hospitalizados realizaram corretamente o uso do inalador do- 
simetrado antes de qualquer explicação. Os pacientes remanescentes aprenderam a usar corretamente o dispositivo após ter sido ensinados. Dos dez pacientes que foram retestados em um período que variou de um a 30 dias, cinco voltaram a fazer o uso do inalador dosimetrado erroneamente.

A heterogeneidade nos métodos utilizados para a avaliação da técnica nos diversos estudos é responsável pela variabilidade na incidência de uso correto de medicação por inalador dosimetrado na literatura. Utilizando a escala de avaliação de técnica de uso de inalador dosimetrado proposto por Manzella et al. ${ }^{(9)}$, Oliveira et al..$^{(10)}$, em outro estudo, demonstraram que houve aumento no número de pacientes que realizavam a técnica totalmente correta (escore $=10$ ), de $4 \%$ para $77 \%$, após um programa educacional que incluiu orientação quanto ao uso do inalador dosimetrado.

É comum na literatura a citação de que pacientes idosos e crianças tendem a apresentar maior dificuldade na aprendizagem; entretanto, faltam estudos direcionados para confirmar essa afirmação. Todd et al. ${ }^{(11)}$ demonstraram que pacientes idosos apresentaram dificuldade em utilizar o inalador dosimetrado; apenas $36 \%$ deles realizaram a técnica de forma totalmente correta; entretanto, os autores não avaliaram pacientes de outras faixas etárias nesse estudo. No nosso estudo, os pacientes com idade abaixo de 15 anos e os acima de 75 anos apresentaram maior incidência de técnica incorreta $(66,7 \%)$, incluindo os erros parcial, intermediário e total, que os pacientes entre essas faixas etárias (47,2\%). Goodman et al.(12) não obtiveram diferença na realização de técnica correta em relação à idade. Nesse estudo, a idade mínima foi de 20 anos e apenas 11 pacientes tinham idade acima de 55 anos.

Em nosso estudo, após a primeira explicação, aproximadamente $50 \%$ dos pacientes aprenderam a usar a medicação de forma totalmente correta. Acreditamos que esse número aumente progressivamente com novas instruções de uso; entretanto, não pudemos determinar isso neste trabalho. Shim e Williams ${ }^{(8)}$, por sua vez, observaram que $20 \%$ dos pacientes falharam em realizar a técnica adequadamente, mesmo após várias explicações.

Além de permitir que a medicação seja administrada com menor dificuldade técnica, a adição das câmaras de expansão aos inaladores dosimetrados oferece inúmeras vantagens sobre seu uso, isoladamente. A deposição faríngea é expressivamente menor $(75 \%$ de redução na dose corporal total), fator importante na administração de corticóide inalatório(1); as câmaras de expansão de grande volume $(700 \mathrm{ml})$ melhoram a distribuição da droga no pulmão, aumentando a deposição pulmonar e o efeito terapêutico(13); paralelamente, resultam em menos efeitos colaterais, devido à menor dose corporal total. Esti- ma-se que, com o uso das câmaras de expansão, a aderência ao tratamento aumente, uma vez que o paciente observa melhora no controle da doença ${ }^{(1,14)}$. Uma vantagem adicional no seu uso é que permite a utilização de medicação por inalador dosimetrado em neonatos e crianças pequenas. Entretanto, apesar de amenizar o problema da pobre coordenação dos pacientes na técnica do uso desses inaladores, persiste a relutância de parte significativa dos pacientes de usar a câmara de expansão em público(13).

Assim, demonstramos neste estudo que, apesar das dificuldades técnicas com o uso do inalador dosimetrado, a maioria dos pacientes aprende a usar corretamente ou quase corretamente a medicação por este dispositivo após uma primeira explicação, desde que exaustivamente ensinados. A insistência no uso da medicação por essa via, e sempre que possivel, associada à câmara de expansão, traz benefícios ao paciente no que concerne ao melhor controle de sua doença e menor incidência de efeitos colaterais.

\section{REFERÊNCIAS}

1. Newhouse MT. Pulmonary drug targeting with aerosols. Principles and clinical applications in adults and children. Am J Asthma Allergy Ped 1993; 7:23-35.

2. Bleecker E. Clinical reality: the safety and efficacy of the world's first CFC-free MDI. Eur Respir Rev 1997;7:37-39.

3. Huchon G. Metered dose inhalers past and present: advantages and limitations. Eur Respir Rev 1997;7:26-28.

4. Tobin MJ, Jenouri G, Danta I, Kim C, Watson H, Sackner MA. Response to bronchodilator drug administration by a new reservoir delivery system and a review of other auxiliary delivery systems. Am Rev Respir Dis 1982;126:670-675.

5. Interiano B, Guntupalli KK. Metered-dose inhalers: do health care providers know what to teach? Arch Intern Med 1993;153:81-85

6. Oliveira MA, Bruno VF, Balini L, Jardim JR, Fernandes ALG. Evaluation of an educational program for asthma control in adults. J Asthma 1997;34:395-403.

7. Epstein SW, Manning CPR, Ashley MJ, Corey PN. Survey of clinical use of pressurized aerosol inhalers. Can Med Assoc J 1979;120:813824.

8. Shim C, Williams MH. The adequacy of inhalation aerosol from canister nebulizers. Am J Med 1980;69:891-894.

9. Manzella BA, Brooks CM, Richards JM, Windsor RN, Soong S, Bailey WC. Assessing the use of metered dose inhalers by adults with asthma. J Asthma 1989;26:223-230

10. Oliveira MA, Farensin SM, Bruno VF, Bittencourt AR, Fernandes ALG. Evaluation of an educational programme for socially deprived asthma patients. Eur Respir J 1999;14:1-7.

11. Todd MA, Basket JJ, Richmond DE. Inhaler devices and the elderly. N Z Med J 1990;103:43-46.

12. Goodman DE, Israel E, Rosenberg M. The influence of age, diagnosis and gender on proper use of metered-dose inhalers. Am J Respir Care 1994;150:1256-1261.

13. Grossman J. The evolution of inhaler technology. J Asthma 1994;31: 55-64.

14. Tinkelman DG, Berkowitz RB, Cole WQ. Aerosols in treatment of asthma. J Asthma 1991;28:243-249. 